\title{
Step Layered Combination of Noninvasive Fibrosis Models Improves Diagnostic Accuracy of Advanced Fibrosis in Nonalcoholic Fatty Liver Disease
}

\author{
Mei Yang ${ }^{1}$, Lina Jiang 1 , Yijin Wang ${ }^{1}, \mathrm{Xi} \mathrm{Li}^{1}$, Guangde Zhou ${ }^{1}$, Zhengsheng Zou², Tao Han ${ }^{3}$, Yuemin $\mathrm{Nan}^{4}$, Fengmin Lu', \\ Jingmin Zhao $^{1}$
}

1) Department of Pathology and Hepatology, the Fifth Medical Center of PLA General Hospital, Beijing 2) The Center for Noninfectious Liver Diseases, the Fifth Medical Center of PLA General Hospital, Beijing 3) Hepatology and Gastroenterology, Tianjin Third Central Hospital, Tianjin 4) Department of Traditional and Western Medical Hepatology, The Third Hospital of Hebei Medical University, Shijiazhaung 5) Department of Microbiology \& Infectious Disease Center, School of Basic Medical Science, Peking University Health Science Center, Beijing,

P. R. China

\begin{abstract}
Background \& Aims: Liver fibrosis is stage-dependently associated with non-alcoholic fatty liver disease (NAFLD) progression. The increased awareness of non-invasive diagnosis has led to the establishment of many fibrosis diagnosis models with various accuracies. We aimed to evaluate the diagnostic performance of nine clinical non-invasive fibrosis models in NAFLD and provide an optimal diagnostic method for advanced fibrosis by step layered combination of non-invasive models.

Methods: 453 consecutive patients with biopsy-proven NAFLD were enrolled from three centers and were divided into study cohort and validation cohort randomly. Aspartate aminotransferase-to-platelet ratio index (APRI), BARD, FiB-4, FibroMeter NAFLD, Forns' Index, Hui model, non-invasive Koeln-Essenindex (NIKEI), S Index and NAFLD fibrosis score (NFS) were calculated. The high area under the receiver operating characteristic curve (AUROC) models were stepwise combined for further diagnosing NAFLD advanced fibrosis.

Results: All models had good performance with high negative predictive value (NPV) and specificity for diagnosing fibrosis, while positive predictive value (PPV) and sensitivity were low. APRI, BARD, FibroMeter NAFLD and NIKEI had higher AUROCs and their step layered combination for diagnosing advanced fibrosis showed high specificity, sensitivity, NPV and PPV up to $89.13 \%, 72.50 \%, 74.36 \%$, and $88.17 \%$, which also performed well in the validation cohort.

Conclusions: APRI, BARD, FibroMeter NAFLD and NIKEI had better diagnostic accuracy, and could be preferred for diagnosing NAFLD fibrosis. The step layered combination of these models performed much better than each single scoring system for diagnosing advanced fibrosis, provides valuable reference for clinical practice and might be a potential substitution of liver biopsy.
\end{abstract}

Key words: NAFLD - liver fibrosis - non-invasive fibrosis model - stepwise combination.

Abbreviations: Alb: albumin; ALP: alkaline phosphatase; ALT: alanine aminotransferases; ApoA1: apolipoprotein-A1; APRI: aspartate aminotransferase-to-platelet ratio index; AST: aspartate aminotransferases; BMI: body mass index; CRN: Clinical Research Network; GGT: $\gamma$-glutamyl transpeptidase; HA: hyaluronic acid; HCC: hepatocellular carcinoma; HDL: high density lipoprotein; IFG: impaired fasting glucose; LDL: low-density lipoprotein; NAFLD: non-alcoholic fatty liver disease; NASH: non-alcoholic steatohepatitis; NFS: NAFLD fibrosis score; NIKEI non-invasive Koeln-Essen-index; NPV: negative predictive value; PLT: platelets; PPV: positive predictive value; PTA: prothrombin time activity; PIIINP: procollagen III N-terminal peptide; Tbil: total bilirubin; TC: total cholesterol; TG: triglyceride; ULN: upper limit of normal.

\section{INTRODUCTION}

Non-alcoholic fatty liver disease (NAFLD) has become the most common chronic liver disease and is estimated to impact at least $17-46 \%$ population, with increasing prevalence in recent years [1]. Non-alcoholic fatty liver disease is a metabolic- stressed liver injury, relating closely to insulin resistance and hereditary susceptibility [2]. It has become a primary indication of liver transplantation and a risk factor leading to an increase in morbidity and mortality from extrahepatic causes (mainly cardiovascular) [3-7]. Due to the global prevalence of metabolic syndrome and the development of potential advanced liver disease, NAFLD has become a vital public health problem around the world.

Non-alcoholic fatty liver disease encompasses a spectrum of diseases from simple fatty liver through non-alcoholic 
steatohepatitis (NASH), to fatty related liver cirrhosis and hepatocellular carcinoma (HCC). The critical features other than steatosis are ballooning degeneration, lobular inflammation and various stages of fibrosis in NASH patients. Liver fibrosis stage, but no other histologic features of NASH, is independently associated with long-term overall mortality, liver transplantation, and liver-related events (i.e. cirrhosis, liver failure and portal hypertension) in NAFLD patients [7]. Advanced liver fibrosis (stage F3 and F4) was the main determinant of not only hepatic but also extrahepatic prognosis in NAFLD patients $[6,7]$. Since the approved treatment regimens for NAFLD are limited, timely diagnosis of NAFLD, especially the detection of advanced fibrosis, has been the key issue for NAFLD patients, and its reliable testing has been of vital clinical significance.

At present, percutaneous liver biopsy is the "gold standard" to evaluate the degree of liver fibrosis. However, it is invasive, has potentially life-threatening complications and might be associated with a degree of inter-observer variability and sampling error [8]. Therefore, establishing a non-invasive diagnostic method for liver fibrosis is urgent for clinical needs. Liver stiffness measurement, a non-invasive fibrosis diagnosis method used in patients with viral liver diseases and alcoholic hepatitis [9] has some restrictions in NAFLD patients who are obese and have a high body mass index (BMI). A large number of biochemical markers related to inflammatory, apoptosis and oxidative stress have also been reported to diagnose liver fibrosis in NAFLD patients [10-12]. However, it is not accurate to predict fibrosis by using only one biochemical marker $[13,14]$. Therefore, the non-invasive diagnostic fibrosis models composed of related clinical and biochemical indicators have become the research focus of non-invasive diagnosis of NAFLD fibrosis.

In recent years, several clinical scoring systems established for viral liver diseases have been applied in diagnosing fibrosis, such as aspartate aminotransferase (AST)-to-platelet ratio index (APRI), BARD score, FIB-4, FibroMeter NAFLD, Forns' Index, Hui model, non-invasive Koeln-Essen-index (NIKEI), $S$ Index [15-23]. NAFLD fibrosis score (NFS) was used to diagnose fibrosis in NAFLD [24]. However, the application effects of these scoring systems for diagnosing fibrosis in NAFLD patients remain unclear or have not been fully validated regarding the diagnostic performance. According to the lack of evidence-based medicine, the American Association for the Study of Liver Diseases (AASLD), European Association for the Study of the Liver (EASL) and Asia-Pacific Alliance of Liver Disease (APALD) guidelines reported that there was no consensus on thresholds or strategies of the non-invasive model for evaluating fibrosis in NAFLD. There is no comprehensive comparison of the accuracy for diagnosing fibrosis in NAFLD by non-invasive models, and further validation is required.

In this study, we evaluated and compared the performance of nine clinical existing non-invasive fibrosis models (APRI, BARD, FIB-4, FibroMeter NAFLD, Forns' Index, Hui model, NIKEI, S Index and NFS) for diagnosing significant fibrosis and advanced fibrosis in a large cohort of patients with biopsyproven NAFLD and verified the diagnostic accuracy of step layered combination of these models, aiming to establish an efficient non-invasive diagnostic method for advanced fibrosis in NAFLD.

\section{METHODS}

\section{Study patients}

The study retrospectively assessed a series of 569 consecutive patients with biopsy-proven NAFLD recruited from September 2012 to December 2017 at the Department of Pathology and Hepatology in Beijing 302 Hospital, Department of Hepatology and Gastroenterology in Tianjin Third Central Hospital and Department of Traditional and Western Medical Hepatology in Third Hospital of Hebei Medical University.

All patients who had increased levels (for more than six months) of liver enzymes, such as aminotransferases and a diffusely hyperechogenic liver at abdominal ultrasonography were considered as candidates for liver biopsy [25]. Patients with normal aminotransferases and hepatomegaly and/or splenomegaly were also potential candidates. The exclusion criteria were: patients with a history of excessive alcoholic consumption (more than $30 \mathrm{~g} /$ day for males and $20 \mathrm{~g} /$ day for females); other etiologies of chronic liver disease (viral hepatitis, drug-induced liver disease, autoimmune hepatitis, primary biliary cirrhosis, genetic hemochromatosis, Wilson disease, etc.). All patients had undergone liver biopsy for the final diagnosis of NAFLD. Enrolled were the patients who had histological changes and clinical criteria for NAFLD.

The study was conducted in accordance with the Declaration of Helsinki. The protocol was approved by the Ethics Committee of the Fifth Medical Center of PLA General Hospital and informed consent was obtained from all patients.

\section{Histological assessment}

In each center, liver biopsy was performed by experienced physicians through percutaneous route with a 16-gauge Hepafix needle under the guidance of B mode ultrasound [26]. All liver biopsy specimens should have at least $15 \mathrm{~mm}$ length and contain 5 complete portal tracts [27]. Liver sections were fixed in $10 \%$ formalin and embedded in paraffin as soon as obtained, and stained with hematoxylin and eosin, silver reticulin, and Masson trichrome routinely. The histological slides of the three centers were collected together and semiquantitatively reevaluated by two experienced pathologists who were blinded to the identity and clinical data of patients. These two pathologists evaluated the slides independently and reevaluated if the results were inconsistent. Histological assessment was according to the NASH Clinical Research Network (CRN) scoring system, in which the fibrosis score is a modification of the Brunt classification score [28]. The fibrosis score system was as follows: F0: no fibrosis; F1a: zone 3 perisinusoidal, delicate; F1b: zone 3 perisinusoidal, dense; F1c: portal only; F2 = F1a or F1b and periportal fibrosis; F3: bridging fibrosis; F4: cirrhosis. Stage F2, F3 and F4 were regarded as significant fibrosis, stage F3 and F4 were considered to indicate advanced fibrosis [29].

Clinical evaluation, laboratory test and non-invasive fibrosis model

Physical examination, physiological and biochemical detection were performed in all NAFLD patients. Fasting blood samples were obtained within one week before the liver biopsy. 
The usual blood variables were necessary for the assessment of model scores and laboratory analyses were assessed, including liver function markers: aspartate and alanine aminotransferases (AST, ALT), AST/ALT radio (calculated as AST divided by ALT), alkaline phosphatase (ALP), $\gamma$-glutamyl transpeptidase (GGT), total bilirubin, cholinesterase, apolipoprotein-A1 (apoA1), prothrombin time activity (PTA), liver fibrosis markers: hyaluronic acid (HA), procollagen III N-terminal peptide (PIIINP), and related biochemical indicators: fasting glucose, platelets, albumin, ferritin, insulin, etc. BMI was calculated as weight $(\mathrm{kg}) /$ height $^{2}\left(\mathrm{~m}^{2}\right)$.

Based on a review of the literature, the following scores were calculated for each NAFLD patient: APRI, BARD score, FIB-4, FibroMeter NAFLD, Forns' Index, Hui model, NIKEI, S Index and NAFLD fibrosis score [15-24]. The detailed formulae used to calculate these model scores are shown in the Supplementary Table I. The cut-off value for each non-invasive fibrosis model was obtained by searching the literature. Age-adjusted cut-offs for NFS and FIB-4 were also considered [30].

\section{Statistical and diagnostic analysis}

Continuous variables were summarized by measures of central tendency and variability as a mean \pm standard deviation, and using percentages and frequency for categorical level parameters. Student $t$-test was used for quantitative variables and Chi square test was used for qualitative variables in order to compare non-significant fibrosis and significant fibrosis, non-advanced fibrosis and advanced fibrosis. Spearman's correlation coefficient was used to estimate the correlation between each non-invasive fibrotic model and stage of NAFLD fibrosis. Receiver operating characteristic (ROC) curve analysis was used to assess the performance of non-invasive fibrosis models in the diagnosis of NAFLD fibrosis by calculating the area under receiver operating characteristic curve (AUROC) with $95 \%$ confidence intervals (95\% CI). The accuracy of sensitivity, specificity, positive predictive value (PPV), and negative predictive value (NPV) for diagnosing NAFLD fibrosis were calculated according to the cut-off value of each non-invasive fibrosis model. The high AUROC models were stepwise combined for the further diagnosis of NAFLD advanced fibrosis.

The statistical computations were carried out using SPSS version 22.0 (SPSS Inc., Chicago, IL, USA) and SAS 9.4 (SAS Institute Inc., Cary, NC, USA). The a error was set at 0.05 (two tailed) and $\mathrm{p}$ value $<0.05$ was considered as statistically significant.

\section{RESULTS}

\section{Patients' characteristics and histology}

A total of 453 NAFLD patients were included in our study. Sixty-eight cases were excluded because of insufficient liver biopsy length and number of complete portal tracts, and other 48 subjects who did not have a full set of clinical data.

The enrolled NAFLD patients included 267 males and 186 females with a mean age of $36.56 \pm 16.72$ years (Table I): 137 patients $(30.24 \%)$ were also diagnosed with type 2 diabetes and 158 cases $(34.88 \%)$ had arterial hypertension. A BMI $>25$ $\mathrm{kg} / \mathrm{m}^{2}$ was present in 218 patients, and 53 patients had BMI >
$30 \mathrm{~kg} / \mathrm{m}^{2}$. Higher ALT and AST levels than the normal upper limit were found in 283 patients $(62.47 \%)$ and 201 patients (44.37\%), respectively.

In the enrolled NAFLD patients, the mean length of the liver biopsy was $17.5 \pm 2.5 \mathrm{~mm}$ and the number of complete portal tracts was $8 \pm 2$. According to the histopathological examination, 199 cases (43.93\%) had a steatosis grade greater than or equal to $66 \%$. A number of 208 patients (45.92\%) had significant fibrosis and 126 patients $(27.81 \%)$ had advanced fibrosis.

All NAFLD patients were divided randomly into a study cohort and validation cohort, and no significant differences were found in the clinical and biochemical indicators between these two cohorts. Table I shows the comparison in clinical and biochemical indicators between the non-advanced fibrosis and the advanced fibrosis groups in the study cohort and validation cohort. Patients with advanced fibrosis had higher levels of ALT, AST, AST/ALT ratio, ALP, insulin and HA, and lower levels of Alb, cholinesterase, urea, ferritin and PTA percent than patients in the non-advanced fibrosis group.

Correlation between each non-invasive fibrotic model and stage of NAFLD fibrosis

Based on the results of liver biopsy, we analyzed the correlation between each non-invasive fibrosis model and stage of NAFLD fibrosis. All nine fibrosis models were correlated with NAFLD liver fibrosis. APRI and BARD were significantly correlated with the degree of NASH fibrosis (rho were 0.638 and $0.524, \mathrm{p}<0.0001)$. FibroMeter NAFLD, Hui model, NIKEI and NFS were moderately correlated with the fibrosis stage (rho $0.484,0.410,0.471$ and 0.437 , respectively). Other models had poor correlation with NAFLD fibrosis (Supplementary Fig. 1, Supplementary Table II).

Diagnostic performance of singular non-invasive model for diagnosing fibrosis

To evaluate the diagnostic accuracy of each non-invasive model for diagnosing NAFLD fibrosis, we performed ROC curve analysis for both significant fibrosis (Supplementary Fig 2, Supplementary Table III) and advanced fibrosis (Fig 1, Table II) in the NAFLD study cohort. APRI, BARD, FibroMeter NAFLD, NIKEI and S Index were significantly different between the advanced fibrosis group and non-advanced fibrosis group ( $\mathrm{p}<0.01$ ). The top four non-invasive models with the highest AUROC values were APRI, FibroMeter NAFLD, NIKEI and BARD. Similar to the performances for diagnosing significant fibrosis, the specificity and NPV for diagnosing advanced fibrosis by single non-invasive model were high, while the sensitivity and PPV were correspondently low. After NFS and FIB- 4 being modified by age, the diagnostic accuracy for NAFLD advanced fibrosis was not changed.

Diagnostic performance of serial step layered combination of non-invasive models for diagnosis of advanced fibrosis

Because of the poor performance of one fold noninvasive model, we finally set up the serial and step layered combination of several fibrosis models for diagnosing advanced fibrosis. According to the higher AUROC, serial step layered combination approach measured APRI for advanced fibrosis 
Table I. Baseline demographic, clinical, and biochemical characteristics of the 453 patients with NAFLD.

\begin{tabular}{|c|c|c|c|c|c|}
\hline \multirow[t]{2}{*}{ Variable $(n=453)$} & \multirow[t]{2}{*}{ All patients } & \multicolumn{2}{|c|}{ Study Cohort $(n=360)$} & \multicolumn{2}{|c|}{ Validation Cohort $(\mathrm{n}=93)$} \\
\hline & & $\begin{array}{c}\text { Stage F0 to F2 } \\
\text { fibrosis }(\mathrm{n}=258)\end{array}$ & $\begin{array}{c}\text { Stage } \mathrm{F} 3 \text { to } \mathrm{F} 4 \\
\text { fibrosis }(\mathrm{n}=102)\end{array}$ & $\begin{array}{l}\text { Stage F0 to F2 } \\
\text { fibrosis }(n=69)\end{array}$ & $\begin{array}{l}\text { Stage F3 to F4 } \\
\text { fibrosis }(\mathrm{n}=24)\end{array}$ \\
\hline Age (years) & $36.56 \pm 16.72$ & $33.35 \pm 15.22$ & $39.19 \pm 17.56$ & $34.12 \pm 19.03$ & $40.33 \pm 17.81$ \\
\hline Male (n, \%) & $267(58.94 \%)$ & $155(60.08 \%)$ & $57(55.88 \%)$ & $41(59.42 \%)$ & $14(58.33 \%)$ \\
\hline BMI $\left(\mathrm{kg} / \mathrm{m}^{2}\right)$ & $25.93 \pm 3.69$ & $25.82 \pm 3.51$ & $26.25 \pm 4.19$ & $26.05 \pm 3.49$ & $26.31 \pm 4.25$ \\
\hline $\operatorname{ALT}(\mathrm{U} / \mathrm{L})^{* *}$ & $135.11 \pm 48.92$ & $127.93 \pm 49.15$ & $173.33 \pm 51.27$ & $128.33 \pm 49.95$ & $174.23 \pm 52.51$ \\
\hline $\operatorname{AST}(\mathrm{U} / \mathrm{L})^{* \ldots \#}$ & $74.12 \pm 35.65$ & $68.88 \pm 27.93$ & $106.74 \pm 49.53$ & $67.50 \pm 26.32$ & $105.91 \pm 48.55$ \\
\hline AST/ALT ratio & $0.69 \pm 0.37$ & $0.63 \pm 0.86$ & $0.78 \pm 0.66$ & $0.62 \pm 0.75$ & $0.77 \pm 0.72$ \\
\hline $\operatorname{ALP}(\mathrm{U} / \mathrm{L})^{* * \# \#}$ & $155.15 \pm 76.82$ & $143.89 \pm 66.91$ & $213.92 \pm 96.43$ & $142.55 \pm 65.31$ & $212.06 \pm 95.83$ \\
\hline GGT (U/L) & $87.66 \pm 52.87$ & $83.85 \pm 49.65$ & $108.91 \pm 62.06$ & $82.29 \pm 50.30$ & $109.53 \pm 63.72$ \\
\hline $\mathrm{TC}(\mathrm{mmol} / \mathrm{L})$ & $4.96 \pm 1.31$ & $4.98 \pm 1.05$ & $4.86 \pm 1.55$ & $5.11 \pm 1.33$ & $4.88 \pm 1.03$ \\
\hline $\mathrm{TG}(\mathrm{mmol} / \mathrm{L})$ & $2.15 \pm 0.93$ & $2.21 \pm 0.99$ & $1.91 \pm 0.99$ & $2.11 \pm 0.87$ & $1.87 \pm 0.76$ \\
\hline $\mathrm{HDL}(\mathrm{mmol} / \mathrm{L})$ & $1.51 \pm 0.75$ & $1.59 \pm 0.86$ & $1.10 \pm 0.56$ & $1.53 \pm 0.90$ & $1.04 \pm 0.71$ \\
\hline $\mathrm{LDL}(\mathrm{mmol} / \mathrm{L})$ & $4.51 \pm 2.09$ & $4.75 \pm 2.31$ & $3.30 \pm 1.22$ & $4.82 \pm 2.59$ & $3.53 \pm 1.96$ \\
\hline apoA1 (g/L) & $1.32 \pm 0.43$ & $1.32 \pm 0.51$ & $1.34 \pm 0.46$ & $1.29 \pm 0.70$ & $1.37 \pm 0.86$ \\
\hline $\operatorname{Alb}(\mathrm{g} / \mathrm{L})^{* * \# \#}$ & $44.97 \pm 4.96$ & $45.89 \pm 4.77$ & $43.55 \pm 4.99$ & $45.25 \pm 6.93$ & $42.01 \pm 5.22$ \\
\hline Tbil (umol/L) & $15.96 \pm 8.01$ & $15.74 \pm 9.95$ & $16.03 \pm 9.25$ & $15.68 \pm 10.21$ & $16.34 \pm 10.44$ \\
\hline Cholinesterase $(\mathrm{U} / \mathrm{L})^{* *}$ & $8935.65 \pm 1788.03$ & $9031.06 \pm 1632.08$ & $8368.36 \pm 2464.81$ & $9005.92 \pm 1608.53$ & $8340.79 \pm 2397.64$ \\
\hline Urea $(\mu \mathrm{mol} / \mathrm{L})^{* \star * \#}$ & $67.09 \pm 19.33$ & $72.05 \pm 19.33$ & $63.11 \pm 13.02$ & $71.56 \pm 20.59$ & $61.77 \pm 15.20$ \\
\hline Ferritin $(\mu \mathrm{mol} / \mathrm{L})$ & $19.91 \pm 6.58$ & $20.02 \pm 6.98$ & $18.57 \pm 7.69$ & $21.57 \pm 7.38$ & $18.96 \pm 6.85$ \\
\hline Fasting glucose $(\mathrm{mmol} / \mathrm{L})$ & $5.36 \pm 1.34$ & $5.34 \pm 1.45$ & $5.43 \pm 1.22$ & $5.25 \pm 2.86$ & $5.52 \pm 1.93$ \\
\hline $\operatorname{PLT}\left(\times 10^{9} \mathrm{~g} / \mathrm{L}\right)$ & $240.12 \pm 66.57$ & $241.05 \pm 64.97$ & $235.01 \pm 83.21$ & $243.66 \pm 62.05$ & $237.83 \pm 82.81$ \\
\hline Insulin $(\mu \mathrm{U} / \mathrm{ml})^{* * \#}$ & $24.93 \pm 17.81$ & $20.88 \pm 11.32$ & $38.22 \pm 26.79$ & $21.53 \pm 12.44$ & $39.05 \pm 29.12$ \\
\hline $\mathrm{HA}(\mu \mathrm{g} / \mathrm{L})^{* * \# \#}$ & $53.67 \pm 49.51$ & $48.90 \pm 39.41$ & $82.73 \pm 84.49$ & $50.12 \pm 38.97$ & $81.90 \pm 79.56$ \\
\hline PTA $(\%)^{* * * \#}$ & $103.08 \pm 15.01$ & $107.03 \pm 14.89$ & $97.91 \pm 17.50$ & $105.85 \pm 17.34$ & $99.23 \pm 19.35$ \\
\hline PIIINP $(\mu \mathrm{g} / \mathrm{L})$ & $6.78 \pm 4.85$ & $6.66 \pm 4.06$ & $7.31 \pm 5.83$ & $6.51 \pm 4.84$ & $7.56 \pm 4.99$ \\
\hline Diabetes & $137(30.24 \%)$ & $66(25.58 \%)$ & $44(43.13 \%)$ & $17(24.64 \%)$ & $10(41.67 \%)$ \\
\hline Hypertension & $158(34.88 \%)$ & $78(30.23 \%)$ & $48(47.06 \%)$ & $22(31.88 \%)$ & $10(41.67 \%)$ \\
\hline
\end{tabular}

Data were expressed in mean \pm SD and as number of cases (\%). p values were standing for comparisons between stage F0 to F2 fibrosis patients and stage F3 to F4 fibrosis patients. ${ }^{\star}$ Between stage F0 to F2 fibrosis and stage F3 to F4 fibrosis in study group, significant at p $<0.05$. \#Between stage F0 to F2 fibrosis and stage F3 to F4 fibrosis in validation group, significant at p $<0.05$. ${ }^{* *}$ Between stage F0 to F2 fibrosis and stage F3 to F4 fibrosis in study group, significant at $\mathrm{p}<0.01$. \#\#Between stage F0 to F2 fibrosis and stage F3 to F4 fibrosis in validation group, significant at $\mathrm{p}<0.05$.

Alb: albumin; ALP: alkaline phosphatase; ALT: alanine aminotransferases; ApoA1: apolipoprotein-A1; AST, aspartate aminotransferases; BMI: body mass index; GGT: $\gamma$-glutamyl transpeptidase; HA: hyaluronic acid; HDL: high density lipoprotein; LDL: low-density lipoprotein; NAFLD: non-alcoholic fatty liver disease; PLT: platelets; PTA:prothrombin time activity; PIIINP: procollagen III N-terminal peptide; Tbil: total bilirubin; TC: total cholesterol; TG: triglyceride.

sequentially followed by BARD, FibroMeter NAFLD and NIKEI (Fig. 2A).

At first, APRI for diagnosing NAFLD advanced fibrosis was tested alone in NAFLD study cohort, and 213 patients (59.17\%) had scores below the cut-off value 0.98 ; 170 patients were non-advanced fibrosis NAFLD, and 43 patients had advanced fibrosis. The remaining 147 patients (40.83\%) had a score higher than 0.98 , of whom 103 had non-advanced fibrosis, 44 had advanced fibrosis. The sensitivity, specificity, PPV and NPV for diagnosis of NAFLD advanced fibrosis were $50.57 \%, 62.27 \%, 29.93 \%$ and $79.81 \%$, respectively.

After NAFLD patients were evaluated by APRI, the BARD, FibroMeter NAFLD and NIKEI were successively added. Among 213 patients with APRI value below 0.98, 179 patients had BARD score less than or equal to 1 point simultaneously; 146 of 179 patients had no advanced fibrosis. In 147 patients with APRI higher than $0.98,103$ had more than or equal to 2 point of BARD score, while 52 patients had no advanced fibrosis and the rest 51 patients had advanced fibrosis. The sensitivity, specificity, PPV and NPV of two step layered combination of APRI and BARD for diagnosing advanced fibrosis were up to $60.71 \%, 73.74 \%, 49.51 \%$ and $81.56 \%$, respectively. The diagnostic accuracy was increased.

FibroMeter NAFLD and NIKEI were added in turn to diagnose following BARD. After FibroMeter NAFLD was added for diagnosis, the sensitivity, specificity, PPV and NPV increased to $70.31 \%, 81.69 \%, 63.38 \%$ and $85.93 \%$, respectively. Finally, when NIKEI was added as the last successive diagnostic step to the layered model combination, the sensitivity, specificity, PPV and NPV improved to $72.50 \%, 89.13 \%, 74.36 \%$ and $88.17 \%$, respectively. The diagnostic performance of serial step layered combination of non-invasive models with 


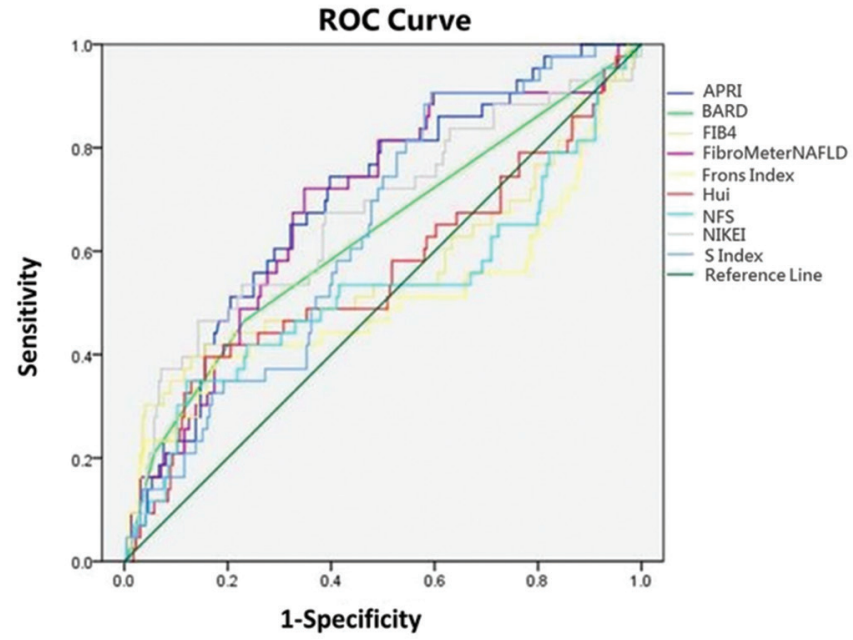

Fig. 1. ROC performance of single non-invasive model for diagnosing NAFLD advanced fibrosis. All enrolled NAFLD patients were diagnosed by each fibrotic model for advanced fibrosis.

APRI, BARD, FibroMeter NAFLD and NIKEI was gradually increasing.

The step layered combination of non-invasive models to diagnose advanced fibrosis was validated in the NAFLD validation cohort (Fig. 2B). In 93 NAFLD validation patients, 55 cases had APRI score below the cut-off value and 38 cases had higher score. The sensitivity, specificity, PPV and NPV for diagnosing NAFLD advanced fibrosis were 52.17\%, 62.86\%, $31.58 \%$ and $80.00 \%$, respectively. After BARD, FibroMeter NAFLD and NIKEI were sequentially added into the diagnosis, 21 cases had non-advanced fibrosis and the sensitivity improved to $72.73 \%$, NPV to $87.50 \%$. Eight patients were diagnosed as advanced fibrosis by the step layered combination of these non-invasive models, and the specificity was $87.50 \%$, and PPV $72.73 \%$.

\section{DISCUSSION}

In the research cohort of 453 NAFLD patients, we compared nine existing non-invasive fibrosis evaluation models to diagnose NAFLD fibrosis and we found that APRI, BARD, FibroMeter NAFLD and NIKEI had the best performance of diagnosing significant fibrosis and advanced fibrosis in NAFLD. We demonstrated that the step layered combination of these four models in diagnosis of advanced fibrosis showed high specificity, sensitivity, NPV and PPV. In the NAFLD validation cohort, this noninvasive stepwise combination test exhibited good stability and accuracy in diagnosing NAFLD advanced fibrosis. The overall efficiency for diagnosing NAFLD advanced fibrosis had been improved by using a step layered combination of APRI, BARD, FibroMeter NAFLD and NIKEI.

Compared with other studies, our 453 NAFLD patients had a younger age, and the equal gender ratio might avoid gender-influencing factors. The characteristics of metabolic syndrome, such as obesity, hypertension, hyperlipidemia, diabetes, were present in our patients. This was consistent with the description of NAFLD associated with the metabolic syndrome in previous studies $[6,31]$. In the histological evaluation, $43.93 \%$ of our patients had hepatic steatosis $\geq$ $66 \%$, and the percentage of steatosis decreased in the patients with F4 fibrosis score. This situation may be due to extensive hepatocyte necrosis, connective tissue hyperplasia and the formation of fibrous septum, leading to the destruction of the hepatic lobule structure and the formation of pseudolobular structure. Meanwhile, lipids may be „burned out” in cirrhotic

Table II. Comparisons of the performances of single non-invasive model for diagnosis of advanced fibrosis in NAFLD study group.

\begin{tabular}{|c|c|c|c|c|c|c|c|}
\hline Model & Cut-off value & $\begin{array}{l}\text { AUROC ( } 95 \% \text { CI) for } \\
\text { advanced fibrosis }\end{array}$ & $P$ value & Sens (\%) & Spec (\%) & PPV (\%) & NPV (\%) \\
\hline APRI & $\begin{array}{l}<0.98: \text { no } \\
\geq 0.98: \text { yes }\end{array}$ & $0.706(0.626,0.785)$ & $0.000^{* *}$ & 50.57 & 60.27 & 29.93 & 79.81 \\
\hline BARD & $\begin{array}{l}<2: \text { no } \\
\geq 2: \text { yes }\end{array}$ & $0.625(0.528,0.723)$ & $0.009^{* *}$ & 46.30 & 75.82 & 25.25 & 88.89 \\
\hline FibroMeter NAFLD & $\begin{array}{l}\leq 0.611: \text { no } \\
\geq 0.715: \text { yes }\end{array}$ & $0.691(0.605,0.777)$ & $0.000^{* *}$ & 59.69 & 86.55 & 43.29 & 87.53 \\
\hline FIB-4 & $\begin{array}{l}<1.3: \text { no } \\
>2.67: \text { yes }\end{array}$ & $0.577(0.467,0.688)$ & $0.009^{* *}$ & 17.95 & 96.30 & 41.18 & 89.04 \\
\hline Forns' Index & $\begin{array}{l}<4.21: \text { no } \\
>6.9: \text { yes }\end{array}$ & $0.515(0.399,0.631)$ & $0.013^{*}$ & 50.00 & 54.75 & 22.86 & 80.33 \\
\hline Hui model & $\begin{array}{l}\leq 0.15: \text { no } \\
>0.5: \text { yes }\end{array}$ & $0.569(0.465,0.673)$ & $0.007^{\star *}$ & 24.39 & 89.08 & 27.78 & 87.24 \\
\hline NIKEI & $\begin{array}{l}\leq 0.0535: \text { no } \\
\geq 0.2294: \text { yes }\end{array}$ & $0.674(0.578,0.770)$ & $0.000^{* *}$ & 21.15 & 95.11 & 42.31 & 87.69 \\
\hline S Index & $\begin{array}{l}<0.1: \text { no } \\
\geq 0.5 \text { : yes }\end{array}$ & $0.649(0.568,0.729)$ & $0.002^{* *}$ & 64.29 & 85.71 & 33.33 & 95.58 \\
\hline NFS & $\begin{array}{l}<-1.455: \text { no } \\
>0.676: \text { yes }\end{array}$ & $0.527(0.417,0.638)$ & $0.008^{\star *}$ & 19.57 & 91.7 & 27.27 & 87.75 \\
\hline
\end{tabular}

Cut-off value: for advanced fibrosis. $\mathrm{P}$ value represented the Delong test. ${ }^{*}$ Significant at $\mathrm{P}<0.05$. ${ }^{*}$ Significant at $\mathrm{P}<0.01$. APRI: aspartate aminotransferase-to-platelet ratio index; AUROC: area under the ROC curve; NAFLD: non-alcoholic fatty liver disease; NFS: NAFLD fibrosis score; NIKEI: non-invasive Koeln-Essen-index; NPV: negative predictive value; PPV: positive predictive value; Sens: sensitivity; Spec: specificity; $95 \%$ CI: 95\% confidence intervals. 


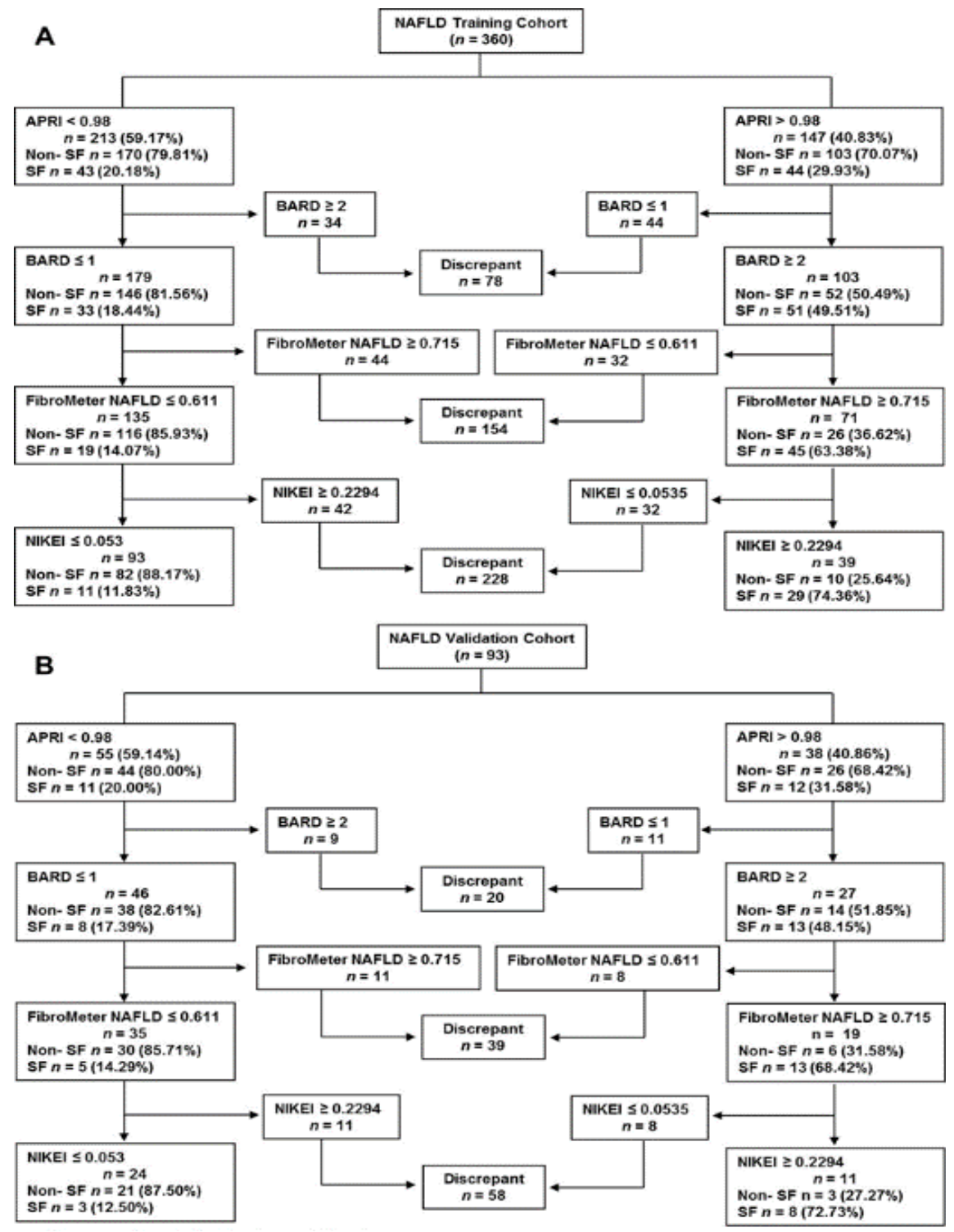

Fig. 2. Diagnostic performance of serial step layered combination of non-invasive models for diagnosing NAFLD advanced fibrosis. Serial step layered combination approach measured APRI for advanced fibrosis sequentially followed by BARD, FibroMeter NAFLD and NIKEI in NAFLD patients in NAFLD study cohort (A) and NAFLD validation cohort (B).

liver [32-34]. In our patients, $37.53 \%$ and $55.63 \%$ cases had normal ALT and AST levels, which was consistent with the existing studies showing that lower levels of ALT and AST levels usually exist in NAFLD patients until the disease progresses to late stages. Thus NAFLD advanced fibrosis could not be diagnosed by ALT and AST levels $[35,36]$.

In our study, all the nine fibrotic models were correlated with the degree of liver fibrosis stage in patients with NAFLD. According to the cut-off values of each model for diagnosing fibrosis in NAFLD, all models had high NPVs and specificity, which were consistent with the existing studies [16, 37, 38], demonstrating that these models could be used to exclude NAFLD significant fibrosis and advanced fibrosis with high accuracy. Nevertheless, none of the models had enough efficacy in PPV, which shows that models were not excellent to detect NAFLD fibrosis. Therefore, all nine non-invasive models showed a greater utility in suggesting the absence of significant fibrosis and advanced fibrosis in NAFLD than in predicting their presence. In our study, the overall performance of the models for predicting advanced fibrosis in NAFLD was poorer than that found in some studies $[36,39]$. This may be caused by the inclusion of younger patients in our enrolled cohort, which might result in a smaller model calculation, affecting the diagnosis of advanced fibrosis. Bertot et al. [40] found that non-invasive fibrosis scoring systems had less diagnostic efficiency at predicting cirrhosis and liver-related outcomes in patients with NAFLD and diabetes. And there were many NAFLD patients with diabetes in our cohort and this may be another reason for the poor performance of the non-invasive fibrosis models in our study. This situation required a large sample of patients for validation and other alternative methods should be used for diagnosis.

According to the performance of diagnosing significant fibrosis and advanced fibrosis by the nine existing non-invasive 
fibrotic models, APRI, BARD, FibroMeter NAFLD and NIKEI had higher AUROC and NPV, and could be preferred in clinical practice for diagnosis of NAFLD fibrosis. Nevertheless, the ideal non-invasive diagnostic models should have high accuracy, including high sensitivity, specificity, NPV and PPV. Our study showed that the singular non-invasive model did not have sufficient accuracy to be used to diagnose NAFLD advanced fibrosis, particularly in view of the severity of this diagnosis. Petta et al. [41] and Demir et al. [42] found that the combination of non-invasive diagnostic models could improve the diagnostic efficiency of NAFLD advanced fibrosis. In our study, step layered combination of APRI, BARD, FibroMeter NAFLD and NIKEI could achieve an overall diagnostic performance improvement in NAFLD advanced fibrosis. The validation experiment showed that this non-invasive step layered test exhibited good stability and accuracy in diagnosing advanced fibrosis in NAFLD patients. At the same time, it was convenient that combination of the scoring systems with existing biochemical serum indicators could be introduced in a pre-designed Excel spreadsheet, which could produce the judgment rapidly without additional costs and avoid invasive liver biopsy in the patients.

Our study has several limitations. Firstly, in order to achieve a homogeneous study cohort, in the enrolled patients we excluded the cases with preexisting diseases and ongoing medical treatment for other diseases, which might result in a selection bias. However, strict inclusion criteria can make the diagnostic judgment more accurate and reduce the influence of other factors. Secondly, our patients were recruited consecutively and the number of patients with NAFLD advanced fibrosis was small. A larger study cohort and more patients with NAFLD advanced fibrosis should be required for validation. Thirdly, liver biopsy was used as the gold standard for evaluation as in all validation studies of non-invasive methods for liver fibrosis, but the sampling bias of liver biopsy was unavoidable. We minimized these biases by an adequate tissue sample length and a number of portal tracts, while the pathological diagnosis of the patients was reevaluated by two experienced pathologists, eliminating the influence of subjective human factors and inter-observer variability for the diagnosis.

\section{CONCLUSIONS}

The APRI, BARD, FibroMeter NAFLD and NIKEI could be preferentially used in clinical practice for the diagnosis of NAFLD fibrosis. The step layered combination of these four fibrosis scores could further improve the diagnostic accuracy of NAFLD advanced fibrosis with good diagnostic performance and might be a potential substitute of liver biopsy.

Conflicts of interest: The authors declare no conflict of interest.

Authors' contributions: M.Y., J.Z., F.J.: study concept and design. T.H., Y.N.: patients' management. M.Y, L.J.: acquisition, analysis and interpretation of data. G.Z., L.J., Z.Z.: histological assessment. M.Y: statistical analysis and drafting of the manuscript. Y.W, X.L.: critical revision of the manuscript. J.Z., F.L.: study supervision. All authors read and approved the final manuscript.
Acknowledgements. This work was supported by grants from the National Nature Science Foundation of China (No. 81673654), the National Science and Technology Major Project (No. 2017ZX10302201, 2017ZX10302201001007) and the Research Found of Capital Medical Development (No. 2014-2-5032). We thank all the patients participating in this study. We thank Ruifang Nie for helpful comments on the earlier draft of the manuscript.

Supplementary material: To access the supplementary material visit the online version of the J Gastrointestin Liver Dis at http://dx.doi. org/10.15403/jgld-420

\section{REFERENCES}

1. Younossi ZM, Blissett D, Blissett R, et al. The economic and clinical burden of nonalcoholic fatty liver disease in the United States and Europe. Hepatology 2016;64:1577-1586. doi:10.1002/hep.28785

2. Katsiki N, Perez-Martinez P, Anagnostis P, Mikhailidis DP, Karagiannis A. Is Nonalcoholic fatty liver disease indeed the hepatic manifestation of metabolic syndrome? Curr Vasc Pharmacol 2018;16:219-227. doi:1 $0.2174 / 1570161115666170621075619$

3. Younossi ZM, Koenig AB, Abdelatif D, Fazel Y, Henry L, Wymer M. Global epidemiology of nonalcoholic fatty liver disease-metaanalytic assessment of prevalence, incidence and outcomes. Hepatology 2016;64:73-84. doi:10.1002/hep.28431

4. Dyson J, Jaques B, Chattopadyhay D, et al. Hepatocellular cancer: the impact of obesity, type 2 diabetes and a multidisciplinary team. J Hepatol 2014;60:110-117. doi:10.1016/j.jhep.2013.08.011

5. Wong RJ, Aguilar M, Cheung R, et al. Nonalcoholic steatohepatitis is the second leading etiology of liver disease among adults awaiting liver transplantation in the United States. Gastroenterology 2015;148:547555. doi:10.1053/j.gastro.2014.11.039

6. Ekstedt M, Hagström H, Nasr P, et al. Fibrosis stage is the strongest predictor for disease-specific mortality in NAFLD after up to 33 years of follow-up. Hepatology 2015;61:1547-1554. doi:10.1002/hep.27368

7. Angulo P, Kleiner DE, Dam-Larsen S, et al. Liver fibrosis, but no other histologic features, associates with long-term outcomes of patients with nonalcoholic fatty liver disease. Gastroenterology 2015;149:389-397. doi:10.1053/j.gastro.2015.04.043

8. Ratziu V, Charlotte F, Heurtier A, et al. Sampling variability of liver biopsy in nonalcoholic fatty liver disease. Gastroenterology 2005;128:1898-1906. doi:10.1053/j.gastro.2005.03.084

9. Ganne-Carrie N, Ziol M, de Ledinghen V, et al. Accuracy of liver stiffness measurement for the diagnosis of cirrhosis in patients with chronic liver diseases. Hepatology 2006;44:1511-1517. doi:10.1002/hep.21420

10. Yoneda M, Mawatari H, Fujita K, et al. High-sensitivity Creactive protein is an independent clinical feature of nonalcoholic steatohepatitis (NASH) and also of the severity of fibrosis in NASH. J Gastroenterol 2007;42:573-582. doi:10.1007/s00535-007-2060-x

11. Wieckowska A, Papouchado BG, Li Z, Lopez R, Zein NN, Feldstein AE. Increased hepatic and circulating interleukin-6 levels in human nonalcoholic steatohepatitis. Am J Gastroenterol 2008;103:1372-1379.

12. Nazal L, Riquelme A, Solı's N, et al. Hypoadiponectinemia and its association with liver fibrosis in morbidly obese patients. Obes Surg 2010;20:1400-1407. doi:10.1007/s11695-009-0051-0

13. Torres DM, Harrison SA. Diagnosis and therapy of nonalcoholic steatohepatitis. Gastroenterology 2008;134:1682-1698. doi:10.1053/j. gastro.2008.02.077 
14. Lewis JR, Mohanty SR. Nonalcoholic fatty liver disease: a review and update. Dig Dis Sci 2010;55:560-578. doi:10.1007/s10620-009-1081-0

15. Wai CT, Greenson JK, Fontana RJ, et al. A simple noninvasive index can predict both significant fibrosis and cirrhosis in patients with chronic hepatitis C. Hepatology 2003;38:518-526. doi:10.1053/jhep.2003.50346

16. Harrison SA, Oliver D, Arnold HL, Gogia S, Neuschwander-Tetri BA. Development and validation of a simple NAFLD clinical scoring system for identifying patients without advanced disease. Gut 2008;57:14411447. doi:10.1136/gut.2007.146019

17. Sterling RK, Lissen E, Clumeck N, et al. APRICOT Clinical Investigators: Development of a simple noninvasive index to predict significant fibrosis in patients with HIV/HCV coinfection. Hepatology 2006;43:1317-1325. doi:10.1002/hep.21178

18. Vallet-Pichard A, Mallet V, Nalpas B, et al. FIB-4: an inexpensive and accurate marker of fibrosis in HCV infection. comparison with liver biopsy and fibrotest. Hepatology 2007;46:32-36. doi:10.1002/hep.21669

19. Calès P, Lainé F, Boursier J, et al. Comparison of blood tests for liver fibrosis specific or not to NAFLD. J Hepatol 2009;50:165-173. doi:10.1016/j.jhep.2008.07.035

20. Forns X, Ampurdanès S, Llovet JM, et al. Identification of chronic hepatitis $\mathrm{C}$ patients without hepatic fibrosis by a simple predictive model. Hepatology 2002;36:986-992. doi:10.1053/jhep.2002.36128

21. Hui AY, Chan HL, Wong VW, et al. Identification of chronic hepatitis B patients without significant liver fibrosis by a simple noninvasive predictive model. Am J Gastroenterol 2005;100:616-623.

22. Demir M, Lang S, Schlattjan M, et al. NIKEI: a new inexpensive and non-invasive scoring system to exclude advanced fibrosis in patients with NAFLD. PLoS One 2013;8:e58360. doi:10.1371/journal. pone.0058360

23. Kun Z, Ruidan Z, Jianchun X, et al. Building a noninvasive diagnostic model based on conventional laboratory markers to predict liver fibrosis. Chinese Hepatology 2008;13:362-367.

24. Angulo P, Hui JM, Marchesini G, et al. The NAFLD fibrosis score: a noninvasive system that identifies liver fibrosis in patients with NAFLD. Hepatology 2007;45:846-854. doi:10.1002/hep.21496

25. Yilmaz Y, Ayyildiz T, Akin H, et al. Gallstone disease does not predict liver histology in nonalcoholic fatty liver disease. Gut Liver 2014;8:313317. doi:10.5009/gnl.2014.8.3.313

26. Wong VW, Wong GL, Choi PC, et al. Disease progression of nonalcoholic fatty liver disease: a prospective study with paired liver biopsies at 3 years. Gut 2010;59:969-974. doi:10.1136/gut.2009.205088

26. Colloredo G, Guido M, Sonzogni A, Leandro G. Impact of liver biopsy size on histological evaluation of chronic viral hepatitis: the smaller the sample, the milder the disease. J Hepatol 2003;39:239-244. doi:10.1016/ S0168-8278(03)00191-0

28. Kleiner DE, Brunt EM, Van Natta M, et al. Design and validation of a histological scoring system for nonalcoholic fatty liver disease. Hepatology 2005;41:1313-1321. doi:10.1002/hep.20701
29. Alkhouri N, McCullough AJ. Noninvasive diagnosis of NASH and liver fibrosis within the spectrum of NAFLD. Gastroenterol Hepatol (N Y). 2012;8:661-668.

30. McPherson S, Hardy T, Dufour JF, et al. Age as a confounding factor for the accurate non-invasive diagnosis of advanced NAFLD fibrosis. Am J Gastroenterol 2017;112:740-751. doi:10.1038/ajg.2016.453

31. Rotman Y, Sanyal AJ. Current and upcoming pharmacotherapy for non-alcoholic fatty liver disease. Gut 2017;66:180-190. doi:10.1136/ gutjnl-2016-312431

32. Abdelmalek M, Ludwig J, Lindor KD. Two cases from the spectrum of nonalcoholic steatohepatitis. J Clin Gastroenterol 1995;20:127-130.

33. Powell EE, Cooksley WG, Hanson R, Searle J, Halliday JW, Powell LW. The natural history of nonalcoholic steatohepatitis: a follow-up study of forty-two patients for up to 21 years. Hepatology 1990;11:74-80. doi:10.1002/hep.1840110114

34. Caldwell SH, Oelsner DH, Iezzoni JC, Hespenheide EE, Battle EH, Driscoll CJ. Cryptogenic cirrhosis: Clinical characterization and risk factors for underlying diasease. Hepatology 1999;29:664-669. doi:10.1002/hep.510290347

35. Harrison SA, Oliver D, Arnold HL, Gogia S, Neuschwander-Tetri BA. Development and validation of a simple NAFLD clinical scoring system for identifying patients without advanced disease. Gut 2008;57:14411447. doi:10.1136/gut.2007.146019

36. Wong VW, Wong GL, Chim AM, et al. Validation of the NAFLD fibrosis score in a Chinese population with low prevalence of advanced fibrosis. Am J Gastroenterol 2008;103:1682-1688.

37. McPherson S, Stewart SF, Henderson E, Burt AD, Day CP. Simple noninvasive fibrosis scoring systems can reliably exclude advanced fibrosis in patients with non-alcoholic fatty liver disease. Gut 2010;59:1265-1269. doi:10.1136/gut.2010.216077

38. Ruffillo G, Fassio E, Alvarez E, et al. Comparison of NAFLD fibrosis score and BARD score in predicting fibrosis in nonalcoholic fatty liver disease. J Hepatol 2011;54:160-163. doi:10.1016/j.jhep.2010.06.028

39. Xun YH, Fan JG, Zang GQ, et al. Suboptimal performance of simple noninvasive tests for advanced fibrosis in Chinese patients with nonalcoholic fatty liver disease. J Dig Dis 2012;13:588-595. doi:10.1111/ j.1751-2980.2012.00631.x

40. Bertot LC, Jeffrey GP, de Boer B, et al. Diabetes impacts prediction of cirrhosis and prognosis by non-invasive fibrosis models in non-alcoholic fatty liver disease. Liver Int 2018;38:1793-1802. doi:10.1111/liv.13739

41. Petta $\mathrm{S}$, Wong VW, Cammà C, et al. Serial combination of non-invasive tools improves the diagnostic accuracy of severe liver fibrosis in patients with NAFLD. Aliment Pharmacol Ther 2017;46:617-627. doi:10.1111/ apt.14219

42. Demir M, Lang S, Nierhoff D, et al. Stepwise combination of simple noninvasive fibrosis scoring systems increases diagnostic accuracy in nonalcoholic fatty liver disease. J Clin Gastroenterol 2013;47:719-726. doi:10.1097/MCG.0b013e3182819a89 


\section{Supplementary Material}

\section{Supplementary Results}

Diagnostic performance of singular non-invasive model for diagnosis of significant fibrosis in NAFLD training cohort

To compare the diagnostic accuracy of the non-invasive models, we performed ROC curve analysis on both significant fibrosis and non-significant fibrosis group (Figure S2). For each model, the AUROC, sensitivity, specificity, PPV and NPV were summarized in NAFLD training cohort (Table S3). APRI, BARD, FibroMeter NAFLD, NIKEI and S Index were significantly distinguished between the significant fibrosis and non-significant fibrosis group $(P<0.01)$. All non-invasive models for significant fibrosis had high specificity and NPV, but the sensitivity and PPV for each model were low. APRI had the best diagnostic effect on significant fibrosis (AUROC 0.713), which

was followed by FibroMeter NAFLD (AUROC 0.677), BARD (AUROC 0.621) and NIKEI (AUROC 0.612). 


\section{Supplementary Tables}

Supplementary Table I. Formulae of non-invasive model scores for diagnosing liver fibrosis

\begin{tabular}{|c|c|}
\hline Model & Equation \\
\hline APRI & APRI $=\left[\right.$ AST level $(/ U L N) /$ platelet counts $\left.\left(10^{9} / \mathrm{L}\right)\right] \times 100$ \\
\hline FibroMeter NAFLD & $\begin{array}{l}\text { AST/ALT ratio at least } 0.8-2 \text { points; } \\
\text { BMI at least } 28 \mathrm{~kg} / \mathrm{m}^{2}-1 \text { point } \\
\text { Presence of diabetes }-1 \text { point. } \\
\text { Score ranges from } 0 \text { to } 4 \text { points. } \\
0.4184 \times \text { glucose }(\mathrm{mmol} / \mathrm{l})+0.0701 \times \mathrm{AST}(\mathrm{UI} / \mathrm{l})+0.0008 \times \text { ferritin } \\
(\mathrm{lg} / \mathrm{l})-0.0102 \times \text { platelet counts }\left(10^{9} / \mathrm{L}\right)-0.0260 \times \mathrm{ALT}(\mathrm{Ul} / \mathrm{l})+ \\
0.0459 \times \text { body weight }(\mathrm{kg})+0.0842 \times \text { age }(\mathrm{yr})+11.6226\end{array}$ \\
\hline FIB-4 & $($ Age $[$ years $] \times$ AST $[I U / L]) /\left(\right.$ platelet counts $\left[10^{9} / L\right] \times \sqrt{\text { ALT }\left[\frac{\mathrm{IU}]}{\mathrm{L}}\right]}$ \\
\hline Forns' Index & $\begin{array}{l}7.811-3.131 \times \ln (\text { platelet counts })(\mathrm{G} / \mathrm{L})+0.781 \times \ln (\mathrm{GGT})(\mathrm{IU} / \mathrm{L})+ \\
3.467 \times \ln (\text { age })(\text { year })-0.014 \times \text { cholesterol }(\mathrm{g} / \mathrm{L})\end{array}$ \\
\hline Hui model & $\begin{array}{l}\exp (3.148+0.167 \times \text { BMI }+0.088 \times \text { bilirubin }[\mu \mathrm{M}]-0.151 \times \text { Alb } \\
\left.[\mathrm{g} / \mathrm{L}]-0.019 \times \text { platelet counts }\left[10^{9} / \mathrm{L}\right]\right) /(1+\exp (3.148+0.167 \times \\
\text { BMI }+0.088 \times \text { bilirubin }[\mu \mathrm{M}]-0.151 \times \text { Alb }[\mathrm{g} / \mathrm{L}]-0.019 \times \text { platelet } \\
\left.\left.\text { counts }\left[10^{9} / \mathrm{L}\right]\right)\right)\end{array}$ \\
\hline NIKEI & $\begin{array}{l}\text { LogitP }=\ln (P / 1-P)=-24.214+0.225 \times \text { age }(\text { year })+0.056 \times A S T \\
{[U / L]+5.044 \times A S T / A L T+3.631 \times \text { Tbil, }[\mathrm{mg} / \mathrm{dL}]}\end{array}$ \\
\hline S Index & $1000 \times \mathrm{GGT}[\mathrm{IU} / \mathrm{L}] /\left(\right.$ platelet counts $\left.\left[10^{9} / \mathrm{L}\right] \times \mathrm{Alb}^{2}[\mathrm{~g} / \mathrm{L}]\right)$ \\
\hline NFS & $\begin{array}{l}-1.675+0.037 \times \text { age }(\text { years })+0.094 \times \text { BMI }(\mathrm{kg} / \mathrm{m} 2)+1.13 \times \\
\text { IFG } / \text { diabetes }(\text { yes }=1, \text { no }=0)+0.99 \times \text { AST } / A L T-0.013 \times \text { platelet } \\
\text { count }\left(\times 10^{9} / \mathrm{L}\right)-0.66 \times \text { Alb }(\mathrm{g} / \mathrm{dL})\end{array}$ \\
\hline
\end{tabular}

Alb, albumin; ALT, alanine aminotransferases; APRI, aspartate aminotransferase-to-platelet ratio index; AST, aspartate aminotransferases; BMI, body mass index; GGT, y-glutamyl transpeptidase; IFG, impaired fasting glucose; NFS, NAFLD fibrosis score; NIKEI, non-invasive Koeln-Essen-index; Tbil, total bilirubin; ULN: upper limit of normal (The lever of AST was $40 \mathrm{IU} / \mathrm{L}$ ) 
Supplementary Table II. Correlation between each non-invasive fibrotic model and stage of NAFLD fibrosis

\begin{tabular}{lcc}
\hline \multicolumn{1}{c}{ Model } & rho & $\boldsymbol{P}$ value \\
\hline APRI & 0.638 & $.000^{* *}$ \\
BARD & 0.524 & $.000^{* *}$ \\
FibroMeter NAFLD & 0.484 & $.000^{* *}$ \\
FIB-4 & 0.384 & $.012^{*}$ \\
Forns' Index & 0.360 & $.000^{* *}$ \\
Hui model & 0.410 & $.009^{* *}$ \\
NIKEI & 0.471 & $.000^{* *}$ \\
S Index & 0.373 & $.003^{* *}$ \\
NFS & 0.437 & $.011^{*}$ \\
\hline
\end{tabular}

"Significant at $P<0.05$.

* Significant at $P<0.01$.

APRI, aspartate aminotransferase-to-platelet ratio index; NAFLD, non-alcoholic fatty liver disease; NFS, NAFLD fibrosis score; NIKEI, non-invasive Koeln-Essenindex;

Supplementary Table III. AUROCs of single non-invasive models to predict significant fibrosis in

NAFLD training cohort.

\begin{tabular}{lllcccc}
\hline \multicolumn{1}{c}{ Model } & $\begin{array}{c}\text { AUROC } \\
(\mathbf{9 5 \%} \text { Cl) for } \\
\text { Significant fibrosis }\end{array}$ & P value & $\begin{array}{c}\text { Sens } \\
\mathbf{( \% )}\end{array}$ & $\begin{array}{c}\text { Spec } \\
\mathbf{( \% )}\end{array}$ & $\begin{array}{c}\text { PPV } \\
\mathbf{( \% )}\end{array}$ & $\begin{array}{c}\text { NPV } \\
\text { (\%) }\end{array}$ \\
\hline APRI & $0.713(0.652,0.773)$ & $.000^{* *}$ & 46.65 & 80.39 & 32.66 & 89.92 \\
BARD & $0.621(0.552,0.691)$ & $.001^{* *}$ & 56.87 & 83.20 & 36.72 & 90.03 \\
FibroMeter NAFLD & $0.677(0.611,0.743)$ & $.000^{* *}$ & 63.27 & 88.90 & 53.33 & 88.51 \\
FIB-4 & $0.538(0.464,0.611)$ & $.009^{* *}$ & 29.72 & 92.17 & 47.06 & 90.25 \\
Forns' Index & $0.459(0.383,0.535)$ & $.008^{* *}$ & 61.52 & 68.93 & 28.75 & 86.96 \\
Hui model & $0.541(0.469,0.614)$ & $.008^{* *}$ & 27.85 & 88.75 & 29.88 & 88.63 \\
NIKEI & $0.612(0.542,0.681)$ & $.001^{* *}$ & 29.57 & 96.01 & 48.65 & 87.90 \\
S Index & $0.590(0.523,0.656)$ & $.011^{*}$ & 66.39 & 83.25 & 39.17 & 93.83 \\
NFS & $0.505(0.430,0.581)$ & $.015^{*}$ & 25.07 & 90.66 & 30.56 & 88.05 \\
\hline PValue
\end{tabular}

$P$ value represented the Delong test.

"Significant at $P<0.05$.

** Significant at $P<0.01$.

APRI, aspartate aminotransferase-to-platelet ratio index; AUROC, area under the ROC curve; NAFLD, non-alcoholic fatty liver disease; NFS, NAFLD fibrosis score; NIKEI, non-invasive KoelnEssen-index; NPV, negative predictive value; PPV, positive predictive value; Sens, sensitivity; Spec, specificity; $95 \% \mathrm{Cl}, 95 \%$ confidence intervals. 


\section{Supplementary Figures}
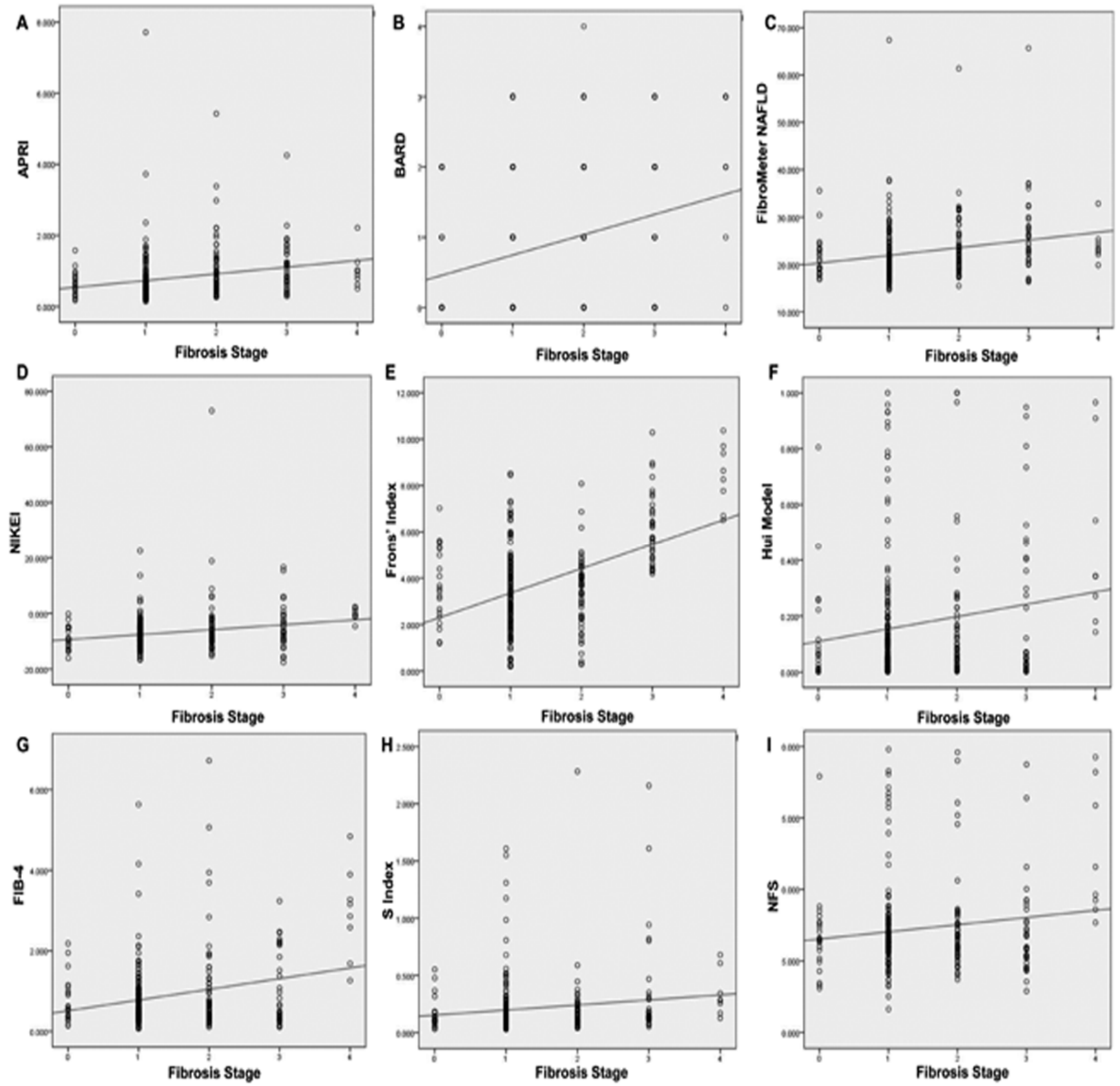

Supplementary Fig. 1. Correlation between each non-invasive fibrotic model and stage of NAFLD fibrosis. All 9 existing fibrotic models in clinical were correlated with NAFLD liver fibrosis. APRI, BARD, FibroMeter NAFLD and NIKEI were moderately correlated with the degree of fibrosis and others were not performance good. 


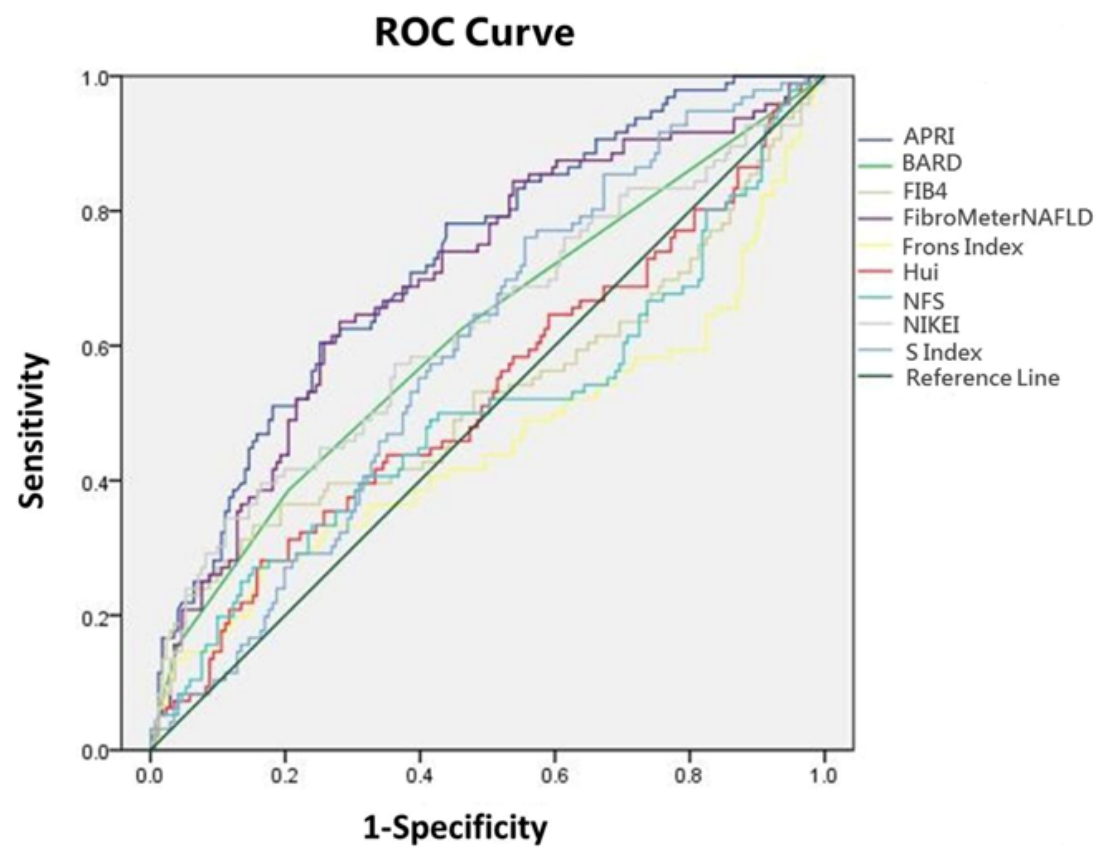

Supplementary Fig. 2. ROC performance of singular non-invasive model for diagnosing NAFLD significant fibrosis. ROC curve analysis were performed for diagnosing significant fibrosis in NAFLD patients using APRI, BARD, FIB-4, FibroMeter NAFLD, Frons' Index, Hui model, NFS, NIKEI and S Index. 